\title{
WHOLE-BODY LYMIPHANGIOSCINTIGRAPHY AND SPECT/CT IN CHILDREN WITH LYMPHATIC COMPLICATIONS AFTER SURGERY FOR COMPLEX CONGENITAL HEART DISEASE
}

\author{
P.H. Kuo, B.J. Barber, R.I. Kylat, S.E. Klewer, S. Behan, S. Lau-Braunhut, \\ M.J. Bernas, L. Moedano, A.D. Bedrick, D.J. Mustacich, M.H. Witte
}

Departments of Medical Imaging, Medicine and Biomedical Engineering (Nuclear Medicine) (PHK), Pediatrics (BJB,RIK,SEK,SL B,ADB), and Surgery (SB,MJB*,LM,DJM,MHW), University of Arizona College of Medicine, Tucson, Arizona, [*Current affiliation: Department of Medical Education, TCU and UNTHSC School of Medicine, Fort Worth, Texas], USA

\section{ABSTRACT}

The number of patients surviving repair of complex congenital heart disease (CCHD) has increased due to improved surgical techniques, post operative management and outpatient care. Likewise, this growing patient population has demonstrated an increasing number and complexity of complications involving the lymphatic system. To evaluate the peripheral and central lymphatic system, whole-body lymphangioscintigraphy (LAS) is considered as the initial imaging evaluation of choice. To date, very few publications exist on the value of lymphatic imaging techniques in infants and small children with lymphatic complications following surgery for congenital heart disease. A retrospective review of medical records from 2008 to 2018 was performed for pediatric patients referred for lymphatic complications after CCHD surgery at an academic medical center. LAS and SPECT/CT was performed using intradermal bipedal injections of Tc $99 \mathrm{~m}$ labeled filtered sulfur colloid, and in some patients also bilateral hand injections, followed by dynamic imaging and whole- body planar imaging typically up to 180 minutes post injection. Clinical decision making and outcomes were recorded. LAS and SPECT/CT were performed without complication in pediatric patients with prior surgery for CCHD. LAS successfully localized various lymphatic abnormalities such as lymphatic obstruction, reflux, and leaks, which were further delineated by SPECT/CT. LAS findings directed further evaluation with more definitive studies, management and prognosis. Five of the ten patients had follow up outcome data -2 years and up to 10 years. LAS and SPECT/CT are safe and effective techniques for the initial evaluation of lymphatic abnormalities in pediatric patients with CCHD. LAS, particularly with further $3 D$ localization by SPECT/CT, provides functional imaging of peripheral and central lymphatic flow and thus provides guidance for medical therapy, non operative interventional management, and surgical therapy for these diverse, debilitating, and often life threatening disorders.

Keywords: whole-body lymphangioscintigraphy, single photon emission computed tomography SPECT/CT, congenital heart disease, Fontan repair, lymphatic complications, chylothorax, plastic bronchitis, protein losing enteropathy

Significant reductions have been achieved in the morbidity and mortality of patients 
undergoing cardiac surgery for complex congenital heart disease (CCHD) $(1,2)$. Although these patients are now surviving well into adulthood, they require close monitoring and ongoing care for complications that often require further interventions (3). This is exemplified in lymphatic complications seen in patients with single ventricle physiology after Fontan surgery which increases central venous pressure with passive systemic venous blood flow to the pulmonary arteries and a functional single ventricular chamber pumping blood to the systemic circulation $(4,5)$. These patients are at risk for multiple complications including increased pressure in the Fontan circuit, collateral vessel formation, and ventricular dysfunction. This unique hemodynamics can lead to decompensation which may produce lymphatic circulatory failure and is one of the reasons for the "failing Fontan" circulation after surgery. Increased lymphatic overload and congestion may lead to ascites, pleural effusion, protein losing enteropathy (PLE), plastic bronchitis (59), and other sites of chylous reflux. The Fontan circulation patients have an elevated central venous pressure, impaired lymphatic pumping capacity, and a morphologically altered thoracic duct, each of which may play a role in the pathogenesis of the lymphatic circulation complications such as protein losing enteropathy, plastic bronchitis, serous effusions, and peripheral edema (10).

Imaging of peripheral and central lymphatic abnormalities is critical for diagnosis, monitoring, and development of a therapeutic plan for CCHD patients. Although symptoms may localize to a specific organ, lymphatic leaks can occur anywhere and thus a whole body approach should be undertaken. One well studied imaging modality for whole-body examination of lymphatic disorders is lymphangioscintigraphy (LAS), which has been recommended as the initial procedure of choice (11-13). LAS is minimally invasive, safe, and technically simple to perform (12). As an imaging modality of whole-body lymphatic architecture, LAS has replaced direct lym- phography, which is more invasive, technically difficult, and carries risks such as oil injury lymphangiopathy and pulmonary oil embolism. LAS provides imaging of the lymphatics over multiple time points as the radiolabeled colloidal tracer migrates through the lymphatic network. Thus, dynamic and static wholebody images and qualitative and quantitative studies can be obtained. Importantly, quantitative LAS analysis permits detection of small changes in lymphatic function over time allowing for comparison or evolution of the disease process or effects of treatment.

Single photon emission computed tomography (SPECT) when combined with computed tomography (CT) to produce hybrid SPECT/CT imaging has the advantage of increased detection and more precise 3D localization of radiotracer thereby improving diagnostic accuracy along with the potential to provide molecular and metabolic information $(14,15)$.

To date, there is a paucity of data on the utility of LAS with or without SPECT/CT in pediatric patients with congenital heart disease. It has been suggested in the literature that the prevalence of lymphatic obstruction and overload is high following single ventricle palliation surgery (5 7). Herein, we examine cases of strongly suspected lymphatic dysfunction and show the utility of LAS and SPECT/ CT for screening and localizing and monitoring lymphatic abnormalities in patients following surgery for CCHD. The aim of our study is to demonstrate the effectiveness of minimally invasive LAS and SPECT/CT in the evaluation of patients following single ventricle palliation for congenital heart disease and in guiding management.

\section{METHODS}

A retrospective review of medical charts was performed for cases of suspected lymphatic disorders in neonates and young children who had surgery for CCHD and underwent LAS studies from 2008 to 2018. CCHD encompasses a variety of anomalies and includes 


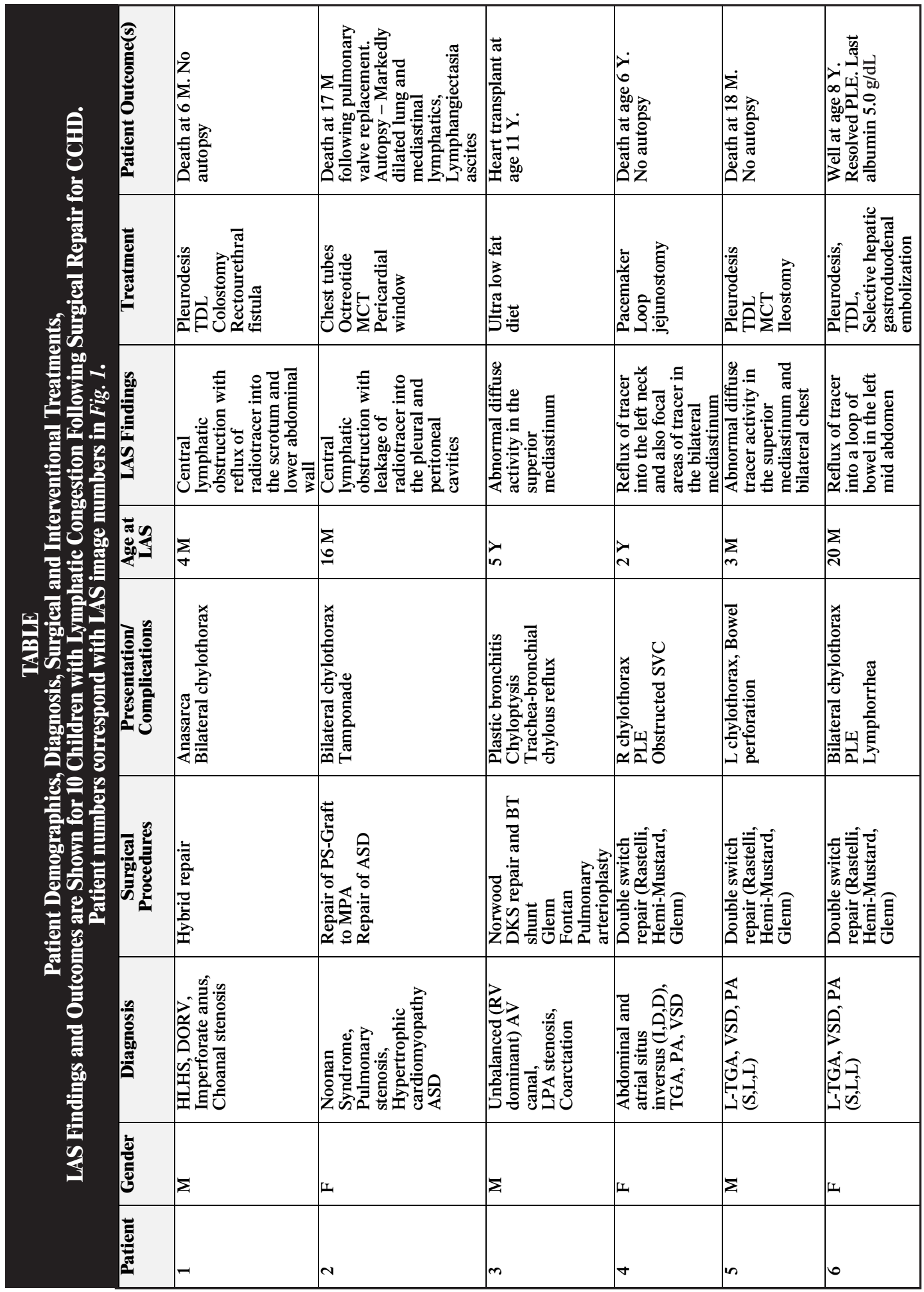




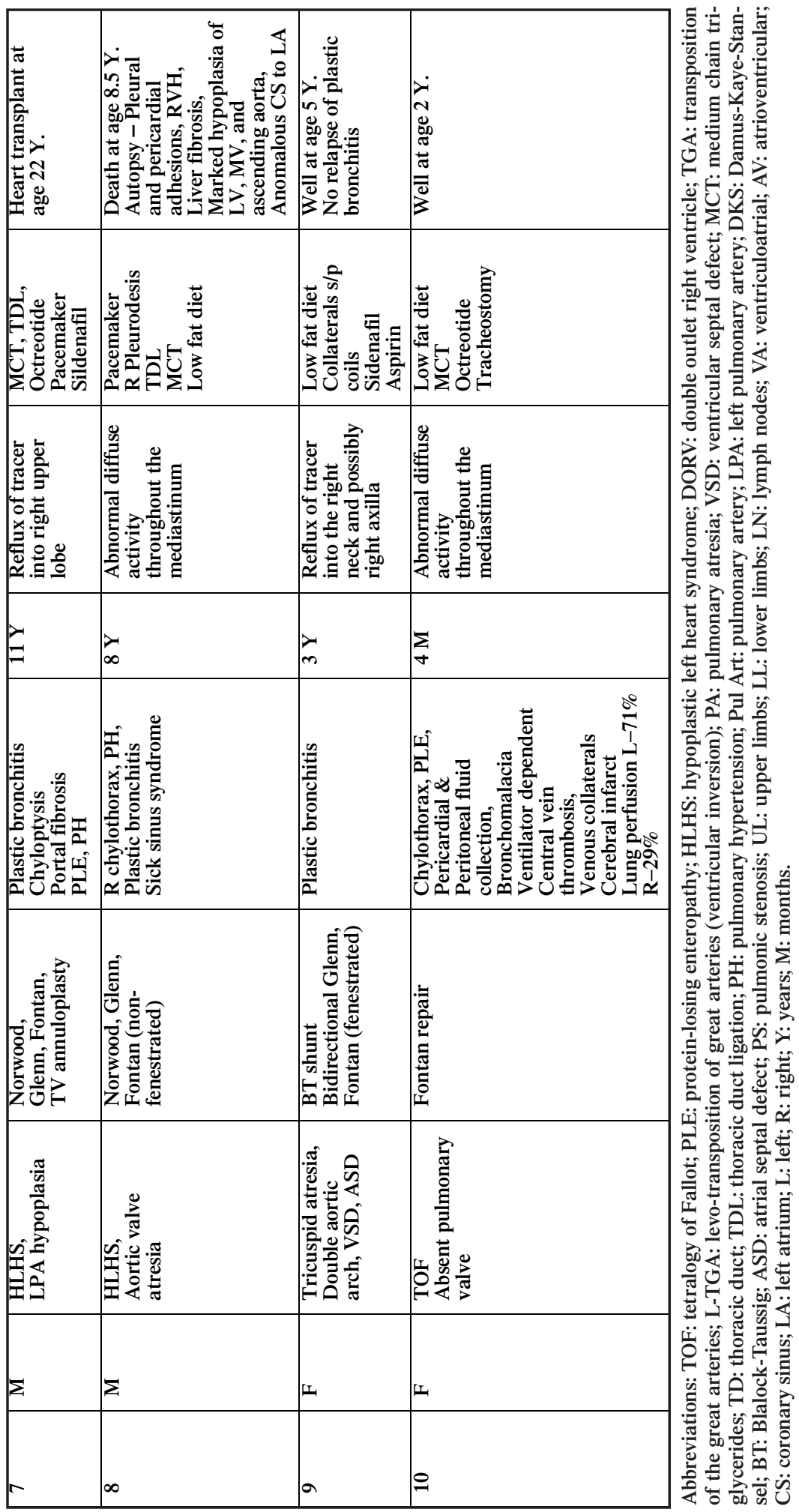


any complex anatomical or physiological lesion as defined by the Bethesda conference (7). Demographic, clinical and outcome data from all CCHD patients, including follow up after LAS study, were retrieved and tabulated. Any complications from LAS or SPECT/CT were also recorded. This study was approved by the Institutional Review Board of the University of Arizona.

LAS was performed following intradermal injection of approximately $500 \mu \mathrm{Ci}(18.5 \mathrm{MBq})$ Tc $99 \mathrm{~m}$ labeled filtered sulfur colloid in $\mathbf{0 . 0 5}$ $\mathrm{ml}$ in the second web space of the feet and/or hands, as needed for lower and upper extremity imaging, respectively. Immediately following injection, dynamic imaging of the lower legs was performed for a period of 15 minutes. Whole-body planar imaging was then typically obtained at $30,60,120$, and 180 minutes post injection. SPECT/CT images were obtained using the General Electric SPECT/CT Discovery 640 equipment usually at 120 minutes following tracer injection but timing varied based upon the timing of optimal LAS images. All images were analyzed by a board certified nuclear medicine physician with knowledge of the clinical history and jointly read with the referring clinician.

\section{RESULTS}

Normal LAS imaging shows prompt, symmetric and uniform migration of the tracer displaying discrete lymphatic channels with visualization of regional lymph nodes 15-20 min post injection, followed later by visualization of liver uptake. Abnormal LAS imaging suggesting lymphatic system pathology includes: increased, decreased or asymmetric number of deep lymphatic vessels; retrograde flow reflecting reflux into superficial valveless lymphatic vessels in the skin or into the intestines, lung or other organs; lymph leak into serous cavities; interrupted, dilated or collateral lymph channels; and decreased number, and asymmetric, or poor visualization of regional nodes (11).

Our retrospective chart review found ten
CCHD patients (ages 3 months to 11 years) who underwent whole-body LAS with clinical follow up. Five of these patients also underwent SPECT/CT examination with 4 concurrently, one at a later time, and one concurrently before and also after lymphatic intervention for PLE by M Itkin and Y Dori at Children's Hospital of Pennsylvania. The specifics of the congenital heart disease and treatment varied (see Table); however, all patients presented with clinical symptoms consistent with various types of lymphatic pathology. Of the ten patients, seven presented with chylothorax, four with plastic bronchitis and four patients had concomitant PLE as detailed in Table.

In all cases, LAS and SPECT/CT were performed without complication and displayed a suspicious site of radiotracer block, spread and/or leakage. In addition, the LAS results correlated with the clinical picture in all ten cases. Importantly, the LAS results, particularly with added SPECT/CT images offering higher spatial resolution and more precise pinpointing of lymphatic leaks, further localized the site and extent of lymphatic disease in all the cases (Table and Figs. 1,2). Accordingly, WBLAS with or without concomitant SPECT/CT provided important guidance for adjusting prognostication and management. All cases were followed up and five of the ten children had long-term follow up.

\section{DISCUSSION}

This retrospective study demonstrates that LAS successfully elucidated the nature and site of lymphatic leak or other abnormality and was consistent with the clinical presentation in all ten CCHD patients that underwent LAS from 2008 to 2018 . The procedure was safe and well tolerated. Furthermore, the use of LAS provided important dynamic information as tracer leakage could be seen early in more proximal lymphatic trunks and then later spread more distally. For example, in case 2 (bilateral chylothorax), tracer activity was seen initially leaking in the mediastinum and then subsequently lateralized to the side 


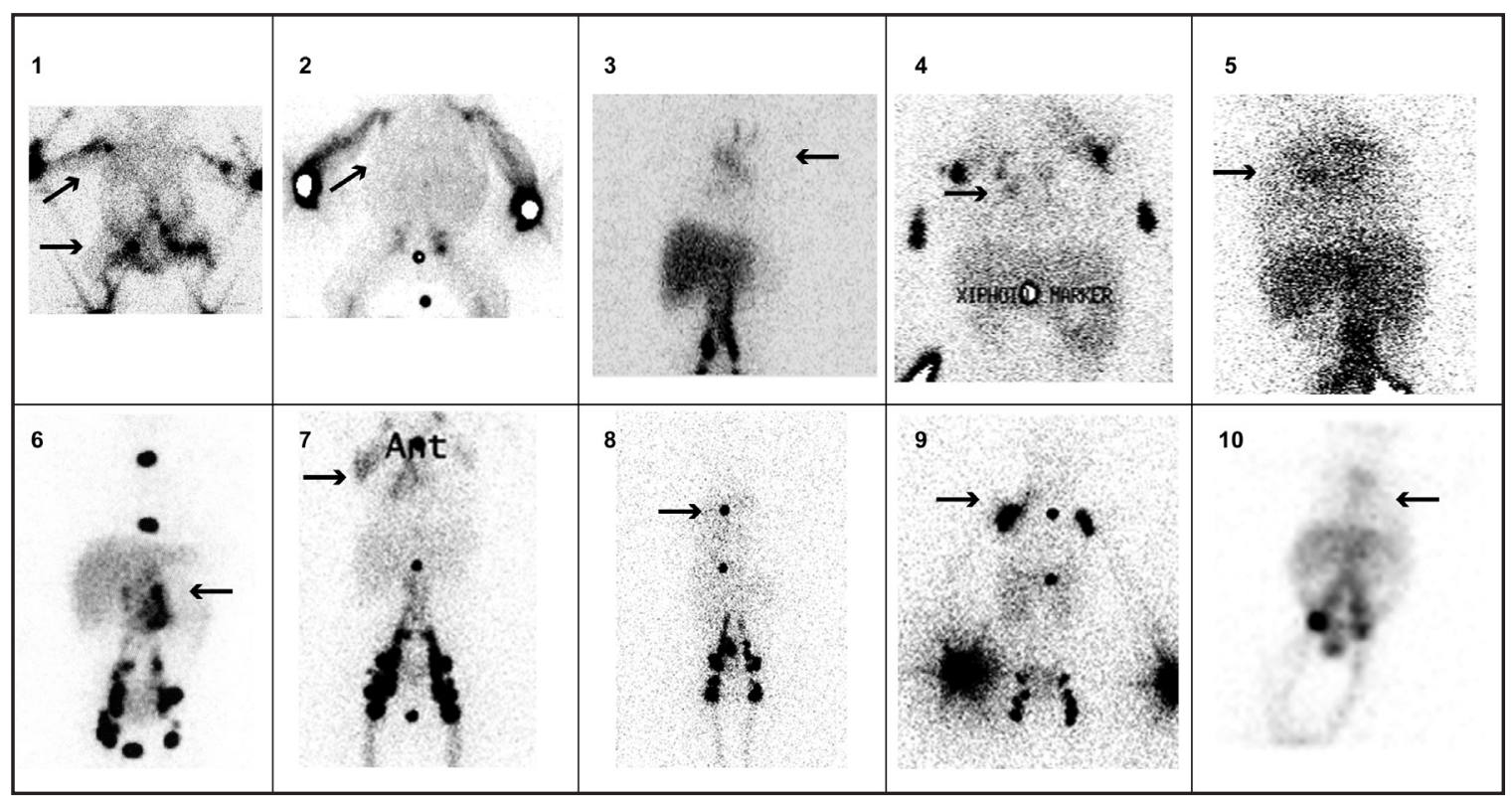

Fig. 1. All patients were injected with tracer in both feet. Patients 1, 2, 4, and 9 also received injections of tracer in their hands to visualize the upper limb lymphatic system. Dense uptake of tracer in the right upper quadrant represents entry of tracer into the liver-blood pool. Arrows point to key areas of abnormal tracer diffusion/reflux. Central round markers identify the knee, pubis, xiphoid, and sternal notch levels in some patients $(2,4-9)$. Panel numbers correspond to patient numbers in Table (see text for further details).

of the chylothorax. In case 6, 3-D SPECT/ CT imaging in follow up to the 2-D LAS was able to precisely localize tracer leakage to the duodenal loop, which explained the protein losing enteropathy, depicting the protein rich lymph fluid leaking directly into the duodenum (image not shown).

The potential benefit of LAS for directing patient management has increased with improvement in surgical management, post operative medical management, interventional MR guided therapy, and other treatments. Lymphatic abnormalities seen in the Fontan circulation result from increased lymph production, central lymph obstruction from elevated central venous pressure, surgical trauma, new lymphatic vessel formation, and associated developmental defects and genetic abnormalities. The lymphatic system has been implicated as a crucial component of the pathophysiology in plastic bronchitis and protein (lymph) losing enteropathy (16-19). Lymphatic embolization procedures are being performed at several academic centers for patients with plastic bronchitis and with PLE with reduction in morbidity and symptoms for affected patients $(5,8,9)$. The use of LAS and SPECT/CT has been helpful as reported in individual case studies in localizing lymphatic pathology and guiding early decision making regarding further imaging, minimally invasive interventions, and/or surgery including interventional and surgical procedures directed at interval thoracic duct decompression without obliteration (19).

By combining the physiologic information from SPECT with the anatomic detail from CT, SPECT/CT images more clearly delineate localization of leaks. WB planar LAS and SPECT/CT provide functional imaging of peripheral and central lymphatic flow and thus offer guidance for medical therapy, non operative interventional management, and surgical therapy for these diverse, debilitating, and often life threatening disorders. SPECT/CT in conjunction with the small dose of radiopharmaceutical for the LAS and the low dose CT portion utilizes a lower dose of radiation than 


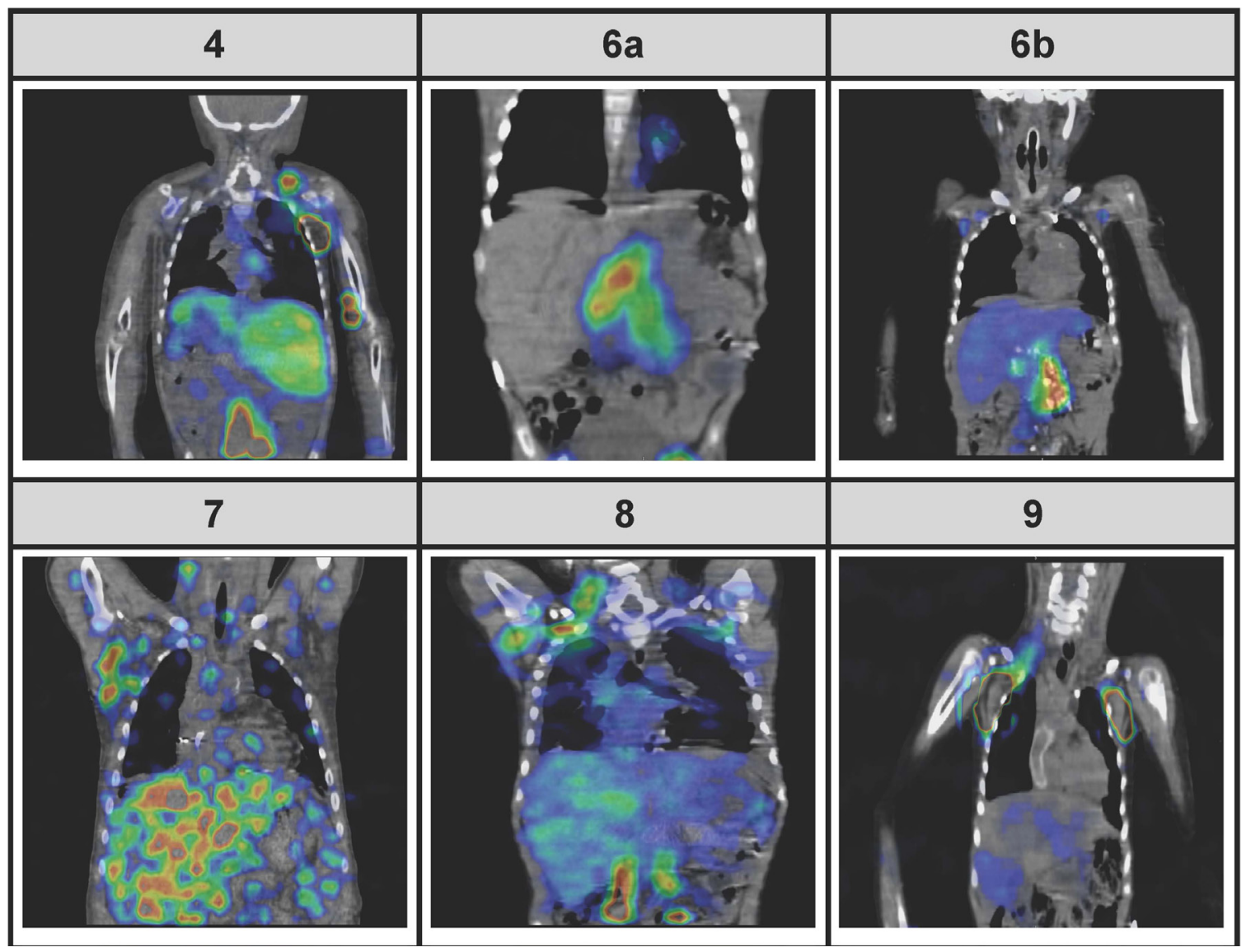

Fig. 2. SPECT/CT images in select children from Fig. 1 (image numbers correspond to patients in Table and Fig. 1). SPECT/CT images show advantageous 3D localization compared to $2 D$ planar WBLAS and also co-registration of SPECT and CT for anatomic correlation. Low radioactivity appears as blue and highest radioactivity as orange with intermediate in yellow. Patient 6 with severe PLE is shown after thoracic duct ligation but before (a) (corresponding to Fig. 1) and also after (b) successful MR lymphangiographic-guided sclerosis of leaking gastroduodenal and hepatic lymphatics performed by M Itkin and Y Dori at Children's Hospital of Pennsylvania with prompt remission of PLE. Note that radiotracer highlighting the duodenal loop in a) is reduced in b) and administered particulate emboli are visualized. Patient 7, who presented with plastic bronchitis (Fig. 1), is shown in the SPECT/CT after thoracic duct ligation to have reduced right tracheobronchial lymphatic leakage but persistent tracer holdup in the right neck and axilla. Patients 7, 8, and 9 show prominent tracer holdup in the right neck and axilla whereas Patient 4 with situs inversus shows tracer holdup in left neck and axilla. Note that mediastinal tracer uptake is more clearly seen than in the corresponding WBLAS in Patients 4, 8, and 9. Prominent tracer uptake in the right upper quadrant (left in patient 4) represents hepatic uptake of sulfur colloid after entry into the venous system.

a diagnostic CT. Thus, although combining the two imaging technologies results in additional radiation exposure, the total radiation dose is less than that of a diagnostic CT and the benefit from such an improved study generally would outweigh the small additional risk from the radiation.

The drawbacks of LAS are few as it requires only an initial intradermal injection in the foot or hand. However, in small children there is risk from sedation, if required, to reduce anxiety and motion artifact. Perhaps the greatest deterrent of the procedure is lack of physician awareness about LAS, since only a small percentage of physicians are aware of the effectiveness and optimal protocols and potential of LAS as the initial study to guide further management. In the ten CCHD 
patients presented here, whole-body LAS abnormalities were noted that were clearly distinct from normal studies. Whether well compensated CCHD patients with few symptoms and complications would show LAS abnormalities following corrective surgery is not known. LAS is more cost effective and lacks the multiple potential contraindications associated with magnetic resonance imaging (MRI), such as cardiac pacemakers, claustrophobia or complications from MR contrast administration.

Awareness of lymphatic complications following congenital heart disease surgery is growing. As studies and publications increase, more physicians are considering lymphatic pathology as a significant cause of morbidity and mortality in these young patients and a variety of new and more refined imaging modalities are under development (20-22). As published data accumulate on the incidence and prevalence of the wide spectrum of lymphatic abnormalities after congenital heart disease surgery, the simple, minimally invasive LAS with dynamic whole-body imaging should be considered in the initial screening work-up. With treatments rapidly improving, the inclusion of this modality in the imaging work up and the subsequent guidance of management it provides, should further improve our capabilities to better the lives of the increasing number of patients living long after surgical correction of complex congenital heart disease.

\section{ACKNOWLEDGMENTS}

This study was supported in part by an intramural Translational Imaging Program Project Stimulus (TIPPS) grant (PHK, MHW) and NIH medical student training grants T35HL07479 (SB, SL-B) and R25HL108831 (LM).
CONFLICT OF INTEREST AND DISCLOSURE

The authors declare no competing financial interests exist.

\section{REFERENCES}

1. Jacobs, JP, JE Mayer Jr, SK Pasquali, et al: The Society of Thoracic Surgeons Congenital Heart Surgery Database: 2018 Update on Outcomes and Quality. Ann. Thorac. Surg. 105 (2018), 680-689.

2. St Louis, JD, H Kurosawa, RA Jonas, et al: The World Database for Pediatric and Congenital Heart Surgery: The Dawn of a New Era of Global Communication and Quality Improvement in Congenital Heart Disease. World J. Pediatr. Congenit. Heart Surg. 8 (2017), 597-599.

3. Reid, GJ, GD Webb, M Barzel, et al: Estimates of life expectancy by adolescents and young adults with congenital heart disease. J. Am. Coll. Cardiol. 48 (2006), 349-55.

4. Gewillig, M, SC Brown: The Fontan circulation after 45 years: Update in physiology. Heart. 102 (2016), 1081-1086.

5. Dori, Y, MS Keller, J Rychik, et al: Successful treatment of plastic bronchitis by selective lymphatic embolization in a Fontan patient. Pediatrics 134 (2014), e590-95.

6. Kreutzer, C, G Kreutzer: The Lymphatic System: The Achilles heel of the Fontan-Kreutzer Circulation. World J. Pediatr. Congenit. Heart Surg. 8( 2017), 613-623.

7. Warnes, CA, R Liberthson, GK Danielson, et al: Task force 1: The changing profile of congenital heart disease in adult life. J. Am. Coll. Cardiol. 37 (2001), 1170-1175.

8. Itkin, M, DA Piccoli, G Nadolski, et al: Protein-losing enteropathy in patients with congenital heart disease. J. Am. Coll. Cardiol. 69 (2017), 2929-2937.

9. Dori, Y, M Itkin: Etiology and new treatment options for patients with plastic bronchitis. J. Thorac. Cardiovasc. Surg. 152 (2016), e4950.

10. Mohanakumar, $\mathrm{S}, \mathrm{N}$ Telinius, B Kelly, et al: Morphology and function of the lymphatic vasculature in patients with a Fontan circulation. Circ. Cardiovasc. Imaging 12 (2019), e008074. 
11. Witte, CL, MH Witte, EC Unger, et al: Advances in imaging of lymph flow disorders. Radiographics 20 (2000), 1697-1719.

12. Turpin, S, R Lambert: Lymphoscintigraphy of chylous anomalies: Chylothorax, chyloperitoneum, chyluria, and lymphangiomatosis - 15-Year experience in a pediatric setting and review of the literature. J. Nucl. Med. Technol. 46 (2018), 123-128.

13. The Diagnosis and Treatment of Peripheral Lymphedema: 2016 Consensus Document of the International Society of Lymphology. Lymphology 49 (2016) 170-184.

14. Israel, $\mathrm{O}, \mathrm{O}$ Pellet, $\mathrm{L}$ Biassoni, et al: Two decades of SPECT/CT - the coming of age of a technology: An updated review of literature evidence. Eur. J. Nucl. Med. Mol. Imaging 46 (2019), 1990-2012.

15. Erba, PA, R Boni, M Sollini, et al: Hybrid imaging of the peripheral lymphatic system. In: Volterrani, D, P Erba, I Carrió, et al (Eds.): Nuclear Medicine Textbook: Methodology and Clinical Applications. Springer, Switzerland. pp 951-975, 2019.

16. Witte, MH: Plastic bronchitis chylous reflux and lymphatic imaging: A continuing story. Lymphology 45 (2012), 44-46.

17. Parikh, K, M Witte, R Samson, et al: Successful treatment of plastic bronchitis with low fat diet and subsequent thoracic duct ligation in child with Fontan physiology. Lymphology 45 (2012), 47-52.
18. Ezmigna, DR, WJ Morgan, MH Witte, et al: Lymphoscintigraphy in plastic bronchitis, a pediatric case report. Pediatr. Pulmonol. 48 (2013), 515-518.

19. James, H, MH Witte, M Bernas, et al: Proposal for prevention or alleviation of protein/ lymph-losing enteropathy (PLE/LLE) after Fontan circulation treatment of univentricular hearts: Restoration of lymph balance with a "lymphatic right-to-left shunt". Lymphology 49 (2016), 114-127.

20. Dori, Y: Novel lymphatic imaging techniques. Tech. Vasc. Interv. Radiol. 19 (2016), 255-261.

21. Guez, D, GJ Nadolski, BA Pukenas, et al: Transhepatic lymphatic embolization of intractable hepatic lymphorrhea. J. Vasc. Interv. Radiol. 25 (2014),149-150.

22. Itkin, M, GJ Nadolski: Modern techniques of lymphangiography and interventions: Current status and future development. Cardiovasc. Intervent. Radiol. 41 (2018), 366-376.

Phillip H. Kuo, MD, PhD

Professor, Medical Imaging, Medicine and

Biomedical Engineering (Nuclear Medicine)

University of Arizona College of Medicine

PO Box 245024

Tucson, AZ 85724-5024

e-mail: pkuo@email.arizona.edu 